\title{
高考化学试题的分析与教学启示一一探究性试题为例
}

\author{
杨懿琳 吴林
}

哈尔滨师范大学

DOI:10.32629/er.v3i3.2546

[摘 要] 化学是一门以实验为基础的自然学科, 实验在帮助学生学好化学具有不可替代的作用,高中化学新课程更加凸显了化学实验在化学教 学中的重要地位和作用。随着新课程改革的不断深入, 学生探究能力的培养越来越受到重视, 本文通过对课程标准与考试大纲进行解析, 分析探 究性试题的主要特点, 同时对近年来新型的探究性试题进行更加深入的分类研究并针对特定的题型进行分析, 进而对相应题型的考试方法进行 了分析,并对探究性试题对教学的启示进行了思考与建议。

[关键词] 高考化学; 探究性试题; 教学实践

在近些年的高考之中, 化学这门学科的试题往往是以实验题的模式来 考量学生的探究性知识的掌握情况。这一类试题主要检验学生在面对试题 内含的信息或材料时, 其阅读与理解信息并与化学的基本知识和技能相结 合的能力, 根据试题所阐述的科学研究的模式, 针对性的提出假设性的构 想, 并严谨地制定一套可行性的探究性实验, 针对实验推理其可能的结果 并加以剖析, 最终提交一个经得起推敲的结论。要能够妥善解答这类探究 性试题, 学生就必须掌握成体系的化学基本知识并能对化学实验有一个基 本的认识; 对一系列的基础化学实验进行熟练的推导, 进而掌握在特定条 件下制定化学实验的设计与分析的技能。对于高中学生而言, 这类题目对 知识储备要求较高, 有着很大的解题难度。探究性试题的材料来源十分之 广泛, 既有来自于科研生产实践活动的实验案例, 亦有来自于教学基础实 验改进案例, 试题重点是考查学生的探究性能力而编写问题。探究性试题 的研究范围十分广阔, 从物质的教材解释以及相应的制备方法, 到相关的 实验装置与方案的设计推导, 都是可以作为问题的取材之处。

\section{1 课标与考纲解读}

《普通高中化学课程标准 (2017年版) 》对实验探究明确提出: 要了解 问题, 设计、评价和优化实验方案, 开展实验活动, 收集实验现象、数据等 相关证据, 根据证据进行分析推理, 以及结论等环节中所需要的方法和策 略。知道如何控制实验设计和实验实施过程中相关变量, 知道如何分析对 实验数据, 能够分析错误的原因, 初步形成定量研究的意识。认识比较、归 纳、分析、综合等方法在分析推理中的应用。在此基础上, 培养学生运用 化学知识和实验方法开展综合项目研究的能力。关于实验技巧: 掌握必要 的实验技能进行实验研究。掌握过滤、蒸馏、萃取、滴定的基本实验操作, 掌握酸度计仪器的使用方法。掌握实验设备组装的基本原则和能力。关于 科学态度: 形成尊重客观事实、细致严谨的科学态度, 具备批判精神和创 新意识。

与2018年相比, 2019年化学考试大纲在第一部分的目标与要求变化不 大, 侧重考生的思想、道德、科学和文化素质要求, 指出 “化学高考以能力 测试为主, 以考生必备知识、关键能力和思维方法为基础, 测试学生的化学 科学素养”。在考试题型中, 可以提高考试题型的思维能力, 更好地反映学 生的学习潜力、灵活运用知识解决实际问题的能力和科学素养。这一变化 也是为即将到来的 2020 年高考搭建了舞台。作为一个热门话题, 探究性实 验问题是相对较固定的。本研究的主要目的是考查学生对化学实验基本方 法和技能的掌握程度、运用化学原理和科学方法的能力, 设计合作项目的 能力以及进行初步研究的能力。利用已知推断未知, 测试学生的学业成绩, 考生必须灵活运用材料性质及制备、分离方法、才能对工业生产中的实际 问题作出合理的预测和解决。

\section{2 探究性试题的命题特点}

2. 1 创设情境及创新

创新是一个民族进步的灵魂, 是一个国家繁荣发展的不竭动力。当 前教育的基本目标是培养具有创新能力的高素质人才。从新课程标准下 各省高考化学实验试题来看, 大多以创设新情境为主题, 这主要体现在 以下三个方面: 第一, 虽然大多数高考题都是以教材为基础, 但在药物的 使用、仪器的开发、实验操作、实验过程等方面进行了优化和创新, 有 时对教材进行几组实验进行小组式考查。第二, 侧重于实验创新的过程, 例如实验原理、实验条件、实验方法和过程的创新, 以及探究问题的大 多数情况是社会生活、科学技术、资源、环境等热点问题。这意味着对 化学知识测试不仅应该是有 “知识性”、“学术性”, 而且应该是 “社会 性” 和 “时代性”。

近几年来, 各省的化学高考, 主要是考查探究性实验和材料制备实验。 这些探究性和制备性实验的问题是全面的, 与理论和实践的紧密相关的, 其中一些还提供新的信息。这就要求考生必须冷静的审视问题, 把所学的 知识和技能, 类比知识、迁移知识、整理知识、综合思维、认真思考结合 起来, 才能正确地回答问题。探究性实验题由于其创新性和灵活性的特点, 继续将成为高考的热门试题。

\section{2 试题结构及分值}

近年来, 在高考试卷中, 探究性试题集中在 $26 、 27$ 题, 分数在 30 分左右, 下设 4 至 6 个小题, 10 个左右填空进行设计, 以 “拼盘” 形式呈现, 题目变化 多样, 知识覆盖面广。

\section{3 高考真题分析}

例题: (2019全国 I 卷) 硫酸铁铵 $\left[\mathrm{NH}_{4} \mathrm{Fe}\left(\mathrm{SO}_{4}\right) \cdot \mathrm{xH}_{2} \mathrm{O}\right]$ 是一种重要铁 盐。为充分利用资源, 变废为宝, 探究采用废铁屑来制备硫酸铁铵, 具体 流程如下:

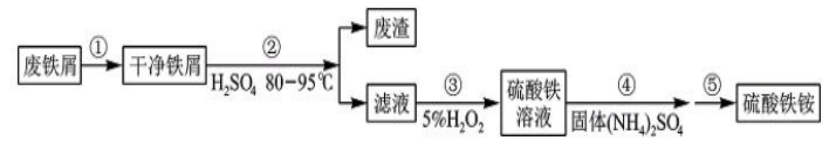

(1) 步骤(1)的目的是去除废铁屑表面的油污, 方法是

(2) 步骤(2)需要加热的目的是 , 温度保持 $80^{\sim} 95^{\circ} \mathrm{C}$, 采用的合适加热方式是—。铁屑中含有少量硫化物, 反应 产生的气体需要净化处理, 合适的装置为 (填标号)。

(3) 步骤(3)中选用足量的 $\mathrm{H}_{2} \mathrm{O}_{2}$, 理由是 分批加入 $\mathrm{H}_{2} \mathrm{O}_{2}$, 同时为了 , 溶液要保持 $\mathrm{pH}$ 小于 0.5 。

(4) 步骤(5)的具体实验操作有 
晶体样品。

(5) 采用热重分析法测定硫酸铁铵晶体样品所含结晶水数, 将样品加 热到 $150{ }^{\circ} \mathrm{C}$ 时, 失掉 1.5 个结晶水, 失重 $5.6 \%$ 。硫酸铁铵晶体的化学式为

解析: (1) 步骤(1)的操作是为了去除铁屑上的油污等杂质, 碱性物质可 以对油污产生水解作用, 故而此处可以利用加热后的碱性的碳酸钠溶液对 其进行清洗。(2) 步骤(2)中 80 至 $95^{\circ} \mathrm{C}$ 的反应条件是为了加快反应的速度; 并且由于选取的反应温度低于水的沸点, 故而可以利用水浴加热来保持反 应装置的温度环境; 此外, 由于铁屑之中含有一定量的硫化物杂质, 在这一 步的反应过程中会产生一定量的硫化氢, 所以需要应用碱液 (即氢氧化钠) 进行吸收, 并且在气体出口处加装漏斗以防倒吸, 综合考虑选择C装置。(3) 步骤(3)是为了将二价铁氧化为三价铁以求接下来进一步的处理, 而选用 $\mathrm{H}_{2} \mathrm{O}_{2}$ 进行反应既可以氧化二价铁, 又不会因此而产生额外的杂质, 故此处可 填入: 将 $\mathrm{Fe}^{2+}$ 全部氧化为 $\mathrm{Fe}^{3+}$, 不引入新的杂质。此外, 又由于 $\mathrm{H}_{2} \mathrm{O}_{2}$ 很容易分 解, 所以为了提高试剂利用率并防止反应生成的三价铁水解, 要分批加入 $\mathrm{H}_{2} \mathrm{O}_{2}$ 试剂, 所以此处可填入: 为了防止 $\mathrm{Fe}^{3+}$ 水解。(4) 需要经过的步骤为: 加 热浓缩、冷却结晶、过滤 (洗涤)。(5) 设硫酸铁铵的化学式为 $\mathrm{NH}_{4} \mathrm{Fe}\left(\mathrm{SO}_{4}\right)$. $\mathrm{xH}_{2} \mathrm{O}$, 其相对分子质量为 $266+18 \mathrm{x}, 1.5$ 个 $\mathrm{H}_{2} \mathrm{O}$ 的相对分子质量为 27 , 则 $27 /(266+18 x)=5.6 \%$, 解得 $x=12$, 则硫酸铁铵的化学式为 $\mathrm{NH}_{4} \mathrm{Fe}\left(\mathrm{SO}_{4}\right) \cdot \mathrm{xH}_{2} \mathrm{O}$ 。

答案：（1)碱煮水洗 (2) 加快反应速率; 热水浴; C (3) 将 $\mathrm{Fe}^{2+}$ 全部氧化 为 $\mathrm{Fe}^{3+}$; 不引入杂质; 防止 $\mathrm{Fe}^{3+}$ 水解 (4) 加热浓缩、冷却结晶、过滤 (洗 涤) (5) $\mathrm{NH}_{4} \mathrm{Fe}\left(\mathrm{SO}_{4}\right)_{2} .12 \mathrm{H}_{2} \mathrm{O}$ 。

分析: 本题是围绕化学实验过程中物质的制备进行考查的综合性探究 试题, 从铁屑制取硫酸铁铵的工艺流程出发, 探讨了实验操作方法、实验原 理、实验操作步骤及注意事项。试题结构合理, 要求能力突出, 整套题目直 接考查了学生的相关探究实验的知识掌握程度, 以及相应的阅读并分析理 解试题的技能, 依据试题条件有序合理地推导出实验结论的能力。这类试 题借助于对实验的合理假设, 通过多种实验条件的创设并变化, 搭建各类 探究性实验相关的问题, 综合地考查考生对于化学基础知识的掌握水平以
及相应的实验探究、分析能力, 满足基于思维和实验的多元化学习能力的 测试要求。

\section{4 探究性试题对教学的启示}

通过对于高考相关试题的分析, 更加确认了学生探究能力的培养的重 要性。利用实际的实验为案例创建探究性的问题进行探究, 以问题导向, 以有效的课堂教学形式来建立探究性的学习实践, 协助学生积极地运用所 学的化学基础知识来解决探究性的问题, 妥善的思考与回答。除了课堂理 论上的学习, 现实的实验实践也是增进学生探究能力的重要方式。要积极 地为学生搭建实验的实践平台, 将所学的化学基础理论运用于实验实践之 中, 通过实践来进一步加深理论认识, 从而全面高效地提升学生解决探究 性问题的能力。并且在实验的过程中, 进一步地提升学生的观察与分析能 力, 养成系统性的假设、分析与解决问题的能力。同时, 实验实践可以引导 学生自主地设计一部分实验操作方案, 能够培养学生数据处理和绘图能力, 以及捕捉有用信息的能力, 使学生的思维更加的灵活、深刻和全面, 实现科 学思维的有效培养, 提高解决问题的能力。

\section{[参考文献]}

[1]中华人民共和国教育部.普通高中化学课程标准(2017版)[S].北京: 人民教育出版社,2018.

[2]普通高中化学课程标准(2017版)解读[S].高等教育出版社,2018.

[3]洪成文 译, 项贤明.教育改革一从启动到成果 [M].教育科学出版 社,2014.

[4]周公度.化学辞典[M].北京:化学工业出版社,2017.

[5]王耀村.科学命题技术研究 [M].杭州:浙江教育出版社,2017.

[6]杜威,王承续译.民主主义教育[M]. 北京: 人民教育出版社,2001.

\section{作者简介：}

杨懿琳(1990--), 女, 汉族, 黑龙江绥化人, 在读硕士研究生, 研究方 向: 学科教学。

吴林(1966--), 男, 汉族, 辽宁锦县人, 工学博士, 副教授, 研究方向: 学科教学。 